\title{
Antiviral Nucleosides-Flu Viruses-Quinolines-COVID-19
}

\author{
Dr Krishnasarma pathy \\ Head QC/QA, IPL Research centre India \\ *Corresponding Author: Dr Krishnasarma pathy, Head QC/QA, IPL Research centre India
}

\begin{abstract}
Hydroquinone as a volatile aromatic hydrocarbon; There is a huge confusion in the public, as well as among politicians and even some scientists, about those two molecules. That confusion ultimately led to people accidently overdosing. Both molecules can be classified as 4-aminoquinolines, and both have antiplasmodial activities, but they remain two different molecules with some different metabolites. Chloroquine has been widely used since the 1950s for prophylactic and curative treatments of malaria. Its extensive use has led to the emergence of chloroquine-resistant strains of Plasmodium falciparum, the parasite responsible for the severest form of malaria. Such strains are also resistant to the structurally related hydroxychloroquine. This article discribes how Guanosine and Hydroxychloroquine is commercially prepared. Also we have recently prepared several processes leading to known antiviral agents starting with guanosine. The processes doveloped by using enzymatic transglycosylation for stavudine (d4T), chemical transpurination for acyclovir and ganciclovir, and novel alkylation for penciclovir and famciclovir.
\end{abstract}

\section{INTRODUCTION}

Chloroquine (Aralen $®$ ) is indicated for the treatment of malaria and extraintestinal amebiasis. Hydroxychloroquine (Plaquenil ${ }^{\circledR}$ ) is also prescribed for the treatment of malaria. It is also used successfully to treat lupus erythematosus and rheumatoid arthritis. In addition, it should be noted that chloroquine, essentially, and hydroxychloroquine, to a lesser extent, have been clinically tested for their antiHIV activity [4,3] We have recently developed several processes leading to known antiviral agents starting with guanosine. The processes involve enzymatic transglycosylation for stavudine (d4T), chemical transpurination for acyclovir and ganciclovir, and novel alkylauon for penciclovir and famciclovir.Firstly, COVID$19^{7}$ and influenza viruses have a similar disease presentation. That is, they both cause respiratory disease, which presents as a wide range of illness from asymptomatic or mild through to severe disease and death. Secondly, both viruses are transmitted by contact, droplets and fomites. As a result, the same public health measures, such as hand hygiene and good respiratory etiquette (coughing into your elbow or into a tissue and immediately disposing of the tissue), are important actions all can take to prevent infection. Human $\mathrm{H} 1 \mathrm{~N} 1^{1}$ pandemic developed from the originally localized Mexican source early in the spring 2009. For the emergency created by the epidemic of «influence of the pigs » in Mexico it was correct not to create alarmismes being victims of a bad information. Cytokine storm should be entioned asone of the key pathogenic events contributing to the overall mortality in substantial portion of patients. If active immunization is assumed to be preventive measure of proven efficacy, clinicians are still in doubt how to treat a complicated course of infection. The possibility that the virus arrives in other parts of the world is real as for all the types of influence virus. In order that a strain has a wide distribution, its antigenic characteristics must ensure that it escapes the neutralization of antibodies of the host and of the surrounding population. So the outbreaks will happen with those strains that have dominant antigens that fit the deficiency, or better, the abscences of antibody in the population.

It seems, in conclusion that the flu virus showes an ability and an aptitude for survival built on the possibility of emergence of new models that allow the virus being confused easily through populations still partly immune to previous antigenic forms. According to this view, the changes in the influenza ${ }^{2}$ A can be designed in single meaning, in the context of a principle and of an evelutionary progress, from Burnet said immunological drift or steering immunology. The antiviral drugs (inhibitors of the neuraminidasis, receptor of the virus surface) should be assumed within 48 hours by the 
appearance of the influence symptoms and for the subjects that have had a close contact with people infected by the flu virus. The vaccination against the influence is the most effective method to prevent the illness. Nowak et al described about air around hydroquinone-containing black-andwhite aqueous developer solutions and found that hydroquinone air concentrations were below the analytical limit of detection surveillance.. In the most recent of these publications, mortality data were reported for 858 men and 21 women engaged in the manufacture and use of hydroquinone from 1942-1990.' Average exposures to hydroquinone ranged from 01-60 $\mathrm{mg} / \mathrm{m}$ ' but for periods of time exposures were as high as $20-35 \mathrm{mg} / \mathrm{m} 3 .{ }^{3}$ In this population, toxic hepatitis and aplastic anaemia have not been associated with hydroquinone exposure. Available data do not support a conclusion that hydroquinone exposure could be associated with hepatitis in the case described by Nowak et al. Bacterial super infections antibiotics treatment, most commonly situated in lower respiratorytract, according to available evidence-basedguidelines on hospital acquired pneumoniamanagement, 3. Low dose cortisol analogues are proper onlywith developed respiratory distress syndromeand together with neuraminidasis inhibitor.High doses are not recommended even asadjuvant therapy and have neither provenefficacy nor safety in this indication.

From the moment that we find the isolation of a new flu virus, we must wait for the preparation of a new specific vaccine that will be ready for the next influence season in Autumn.Combining a biological process ${ }^{5,6}$ for the production of guanosine 1 and a direct phosphorylation process of the unprotected nucleoside, an industrial process for 5'-GMP was commercialized in Japan and India. Having 1available in quantity at a reasonable cost, we envisioned establishing a practical process for manufacturing the major known antiviral nucleosides such as stavudine 2, acyclovir 3, ganciclovir 4, penciclovir 5 and famciclovir 6. In order to realize this, we carried out extensive studies on enzymatic transglycosylation, chemical transpurination and novel N9-selective alkylation of guanine derivatives.
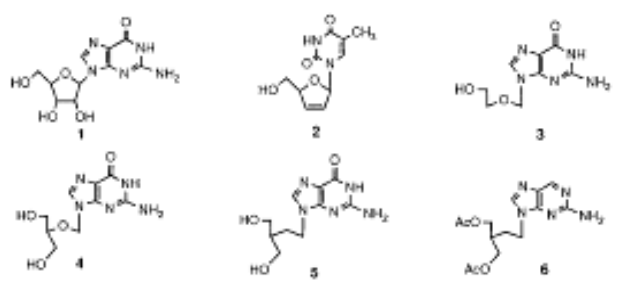

5'-Guanylic acid (Guanosine 5'-monophosphate, 5 '-GMP) is one of the nucleotides widely distributed in nature and has been isolated from hydrolyzates of yeast RNA in 1911 (Ref. 15). Since the disodium salt of 5'-GMP was recognized as a good flavor enhancer (Ref. 16) and later found to be a major flavor component of shiitake mushroom (lentinus edodes sing.), much attention has been paid to the industrial process for 5'-GMP.

An acute respiratory disease, caused by a novel coronavirus (SARS-CoV-2, previously known as 2019-nCoV), the coronavirus disease 2019 (COVID-19) ${ }^{8}$ has spread throughout China and received worldwide attention. On 30 January 2020, World Health Organization (WHO) officially declared the COVID-19 epidemic as a public health emergency of international concern. The emergence of SARS-CoV-2, since the severe acute respiratory syndrome coronavirus (SARS-CoV) in 2002 and Middle East respiratory syndrome coronavirus (MERS$\mathrm{CoV}$ ) in 2012, marked the third introduction of a highly pathogenic and large-scale epidemic coronavirus in to the human population in the twenty-first century. As of 30 March 2020, a total of 4,87,137 confirmed cases globally, deaths (7\%) had been reported by WHO. Meanwhile, several independent research groups have identified that SARS-CoV-2 belongs to $\beta$ coronavirus, with highly identical genome to bat coronavirus, pointing to bat as the natural host. The novel coronavirus uses the same receptor, angiotensin-converting enzyme 2 (ACE2) as that for SARS-CoV, and mainly spreads through the respiratory tract. Importantly, increasingly evidence showed sustained human-to-human transmission, along with many exported cases across the globe.Here we wish to report the practical syntheses of antiviral nucleosides starting with 1.Synthesis of stavudine $(\mathrm{d} 4 \mathrm{~T}, 2)$ via enzymatic transglycosylation. Enzymatic transglycosylation reactions were extensively studied by Utagawa $\boldsymbol{e t} \boldsymbol{a l}$. in these laboratories (Ref. 17). Utilizing this enzymatic process, AraA (adenine arabinoside, 9) has been commercially produced from Ara-U (uracil arabinoside, 8) as a sugar donor and adenine as a sugar acceptor (eq. 1, Ref. 18). Enzymatic transglycosylation was also applicable to the synthesis of 2',3'-dideoxynucleosides, i.e. ddU 10 was converted into ddA lla $(\mathrm{R}=\mathrm{NH} 2)$ and ddI llb ( $\mathrm{R}=\mathrm{OH})$ (eq. 2, REF. 19). 

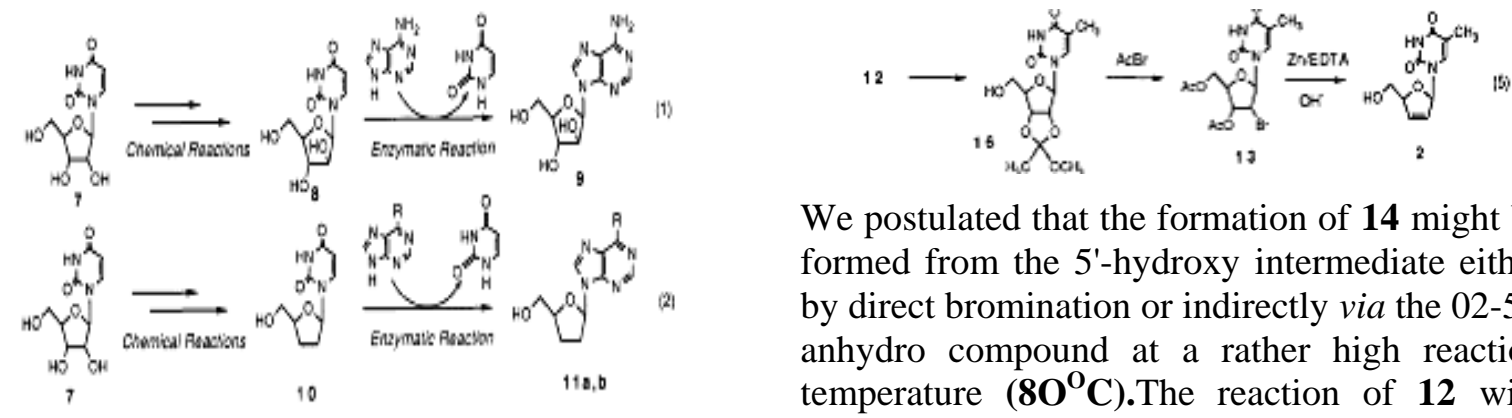

We postulated that the formation of $\mathbf{1 4}$ might be formed from the 5'-hydroxy intermediate either by direct bromination or indirectly via the 02-5Ianhydro compound at a rather high reaction temperature $\left(\mathbf{8 0}^{\circ} \mathrm{C}\right)$. The reaction of $\mathbf{1 2}$ with acetyl bromide apparently produces water in sitii which may prevent acetylation of the 5'position and consequently cause the formation of 14. Based on this hypothesis, we investigated an alternative indirect route to the synthesis of 13 which consists of the methoxyethlidenation of 12 followed by acetoxybromination under milder conditions. We anticipated that the reaction of the methoxyethylidene compound $\mathbf{1 6}$ with acetyl bromide dose not produce water so that the formation of $\mathbf{1 4}$ should be diminished. 12 was reacted with trimethyl orthoacetate in acetic acid. The rnethoxyethylidene derivative 16 was isolated from the reaction mixture although it could be used for further reaction simply after concentration. The residue was then reacted with acetyl bromide and/or $\mathrm{HBr} / \mathrm{AcOH}$ at a lower temperature $\left(5 \mathrm{O}^{\circ} \mathrm{C}\right)$. As a result, 13 was obtained in a high yield and the formation of 14 was successfully reduced $(0-1.3 \%)$. The best result $(90 \%)$ was obtained by the application of Chu's conditions $(\mathrm{AcBr}$ and $\mathrm{HBr} / \mathrm{AcOH})$ to the methoxyethylidene derivative 16. Several methods for reductive pelimination of bromoacetate nucleosides have been reported (Ref. 23 Considering the question of scaling up, we adopted a zinc mediated reductive elimination process due to the mildness of the reaction and the ease of waste treatment. The reductive elimination proceeded smoothly by adding zinc powder to $\mathbf{1 3}$ and afforded more than a $90 \%$ yield of 5'-O-acetyld4T. After completion of the reaction, the mixture became homogeneous and was treated with aqueous EDTA sodium salt yo remove zinc. The d4T acetate thus obtained was then hydrolyzed with aqueous $\mathrm{NaOH}$, purified with a resin and crystallized from water to give d4T, 2 in a $72 \%$ yield from $\mathbf{1 3}$ (eq. 5, Ref. 24). The four step reaction sequence described above gave $\mathbf{2}$ in a $65 \%$ overall yield from $\mathbf{1 2}$ as opposed to $45 \%$ overall yield using Mansuri's direct acetoxybromination approach.

The clinical symptoms of COVID- $19^{10}$ patients include fever, cough, fatigue and a small population of patients appeared gastro-intestinal infection symptoms. The elderly and people 
with underlying diseases are susceptible to infection and prone to serious outcomes, which may be associated with acute respiratory distress syndrome (ARDS) and cytokine storm. Currently, there are few specific antiviral strategies, but several potent candidates of antivirals and repurposed drugs are under urgent investigation. In this review, we summarized the latest research progress of the epidemiology, pathogenesis, and clinical characteristics of COVID-19, and discussed the current treatment and scientific advancements to combat the epidemic novel coronavirus

${ }^{1}$ The history of flu viruses teaches that the influence has origin from animals birds, generically aquatic, then transfered to man through the leap into pigs. The promiscuity of the herds, as it is in use in Asia, determines this transition and then the spread. The Spanish influence (1918, H1N1), the one from Asia (1957, H2N2), that of Hong Kong (1968, H3N2) and so on have had this origin The strains common in some years may have also relations with those of other years.

The persons mostly old people have antibodies directed towards the antigens more important of the strains with which they were in contact. With the progress of the age it is a broader spectrum immunity that is reflected in antibodies polyvalent made through the contact with many antigens primary and secondary present in strains that they meet during the following years. But each contact following with a flu virus of type A involves not only specific antibodies, but also an increase in those directed towards the strain responsible for the first flu infection of the subject (phenomenon of Davenport or doctrine of original sin). In this way, the immunization to a particular strain, spread in a certain period, involves progressively increasing difficulty in its further distribution and creates the selective advantage, for some variant of the virus, to multiply and spread.

Synthesis of Acyclovir 3 via Chemical Transpurination followed by Isomerization.

Acyclovir $\mathbf{3}$ is an orally active acyclic nucleoside with inhibitory activity against herpes viruses which is used widely throughout the world. Several methods for the synthesis of acyclovir have already been reported (Ref. 25 . Amongst these, one of the most convenient and efficient methods is the one which involves the condensation reaction of $\mathrm{N}, \mathrm{N}$ '-diacetylguanine 17 with (2-acetoxyethoxy)methyl acetate in the presence of p-toluenesulfonic acid (eq. 6, Ref. 26). The drawbacks in this procedure are the accompanying formation of considerable amounts of N7-isomer 19 and the difficulty of its separation by an industrial process. Boryski and Golankiewicz studied the transpurination of tetraacetylguanosine $\mathbf{2 0}$ and obtained 9-(2acetoxyethoxymethyl)-N -acetylguanine 18 and its 7 -isomer 19 in a ratio of 9/7 $=47 / 39$ (eq. Ref 7). It is of interest that the same authors observed that 19 isolated by chromatography could be transformed in a similar ratio $(9 / 7=55 / 45)$ of the mixture applying thermal isomerization in chlorobenzene at 23OOC (REF. 27). In order to establish a more economical process for the synthesis of acyclovir $\mathbf{3}$, we intended to use guanosine $\mathbf{1}$ as a starting material and find out the conditions leading to the exclusive formation of the desired 9-isomer 18. After several unsuccessful attempts, we examined the reaction of $\mathbf{1}$ with (2acetoxyethoxy)methyl acetate in acetic anhydride as a solvent and in the presence of ptoluenesulfonic acid as a catalyst. As a result, it was found that diacetylacyclovir (18 and 19) was obtained in a satisfactory yield $(86 \%)$ as a mixture of 9/7 isomers in a ratio of 1.45 . Surprisingly, the ratio was much improved to 17.1, after concentrating the reaction mixture and then heating the residue at $100^{\circ} \mathrm{C}$ under reduced pressure for 24 hours. The isomerization conditions were considerably milder than those reported by Boryski $\boldsymbol{e t}$ al

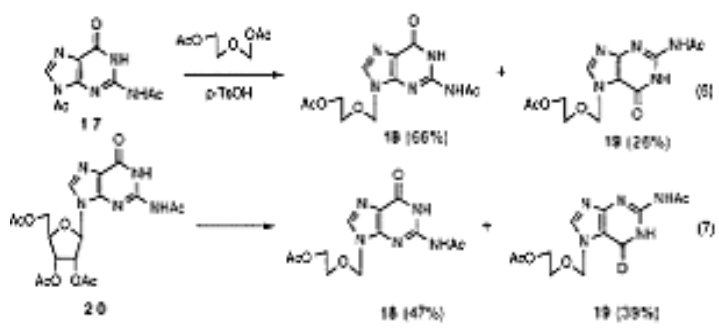

By adding an appropriate solvent such as ethyl acetate and acetonitrile to the residue, almost pure $18(9 \mathrm{R}=120)$ was obtained in an $86 \%$ yield from 1

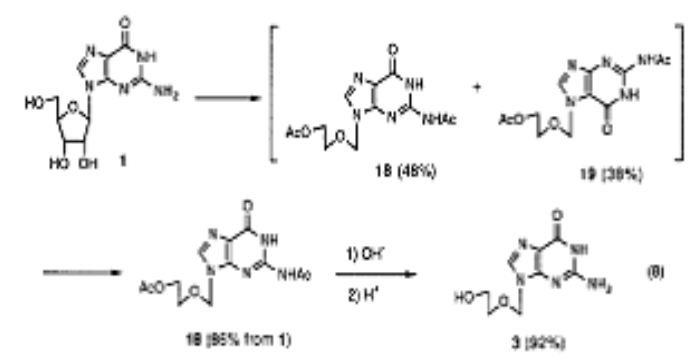

Since the catalyst in the synthesis of the acyclic sugar moiety could be the same as that used in 
the uanspurination, the one pot reaction of 1 with 1,3-dioxolane and acetic anhydride in the presence of acid catalyst gave similar results. Treating 18with aqueous $\mathrm{NaOH}$ followed by neutralization with hydrochloric acid gave acyclovir 3 in a 92\% yield to a high purity, which means that the overall yield of $\mathbf{3}$ from 1was $78 \%$ (eq. 8 , Ref. 26). It was confirmed that the method is also satisfactorily applicable to the synthesis of ganciclovir4 which is used for clinical treatment of cytomegalovirus infection in immunodeficient patients.

\section{Novel Regioselective Synthesis of 9-Alkylated Guanine Derivatives.}

Since the first report describing that acyclovir has potent activity against herpes virus, acyclic nucleoside analogs have been the focus of much attention in the development of new antiviral therapeutic agents (Ref. 28). Among them, penciclovir $\mathbf{5}$ and its orally active form famciclovir 6 were recently approved as new types of anti-hepesvims agents. Since 6 has a 2aminopurine nucleus which is easily transformed from the guanine nucleus, we first attempted to develop new methodology for the synthesis of 9-alkylated guanine derivatives, in particular that of penciclovir 5. Although the structure of $\mathbf{5}$ is closely related to ganciclovir $\mathbf{4}$, oxygen in the side chain of $\mathbf{4}$ is replaced with methylene in 5. Accordingly, the transpurination- isomerization method employed for the synthesis of acyclovir 3 and ganciclovir 4 by us can not be applied to the synthesis of 5 . In order to obtain acyclic guanine nucleoside derivatives, 2-amino-6-substituted purine 21 (often 2-amino-6-chloro-: $\mathrm{R}=\mathrm{C} 1$ ) is commonly used for alkylation (Ref. 29). A problem associated with this method is that the alkylation is rarely regiospesific, and although the desired N9 isomer 23 is the major product, the N7 isomer $\mathbf{2 4}$ is also formed to a significant extent (eq. 9). From an industrial viewpoint, it should be noted that there is another serious disadvantage using 2-amino-6-halo- purine 21 $(\mathrm{R}=$ halogen) as a starting material. Namely, the synthesis of 21 is not so efficient because tedious operational steps and a rather low yield render the cost high.

Guanosine 1can be considered as N9-protected guanine. It is well known that $\mathbf{1}$ is alkylated at the 7-position under neutral conditions and that the ribose moiety is then readily removed by acid treatment to give N7-alkylguanine. We thought that it might be possible to obtain N9alkylated guanine exclusively, if the alkylation of N7-alkylguanine selectively takes place at the N9-position and then the N7-alkyl group is selectively removed. Thus, N7-benzylguanine 25 was selected as our starting material since the N7-benzyl group could be selectively removed by catalytic hydrogenolysis after the alkylation reaction. 25 was obtained in more than a 90\% yield from 1 according to the modification of reported procedure (eq.10, Ref. 30).

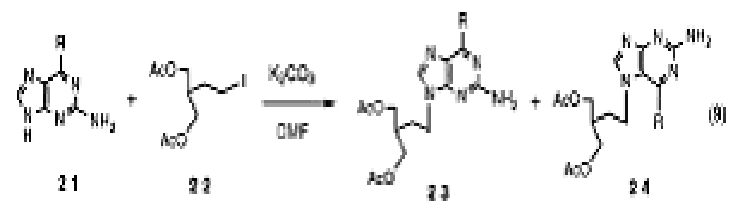

The alkylation of 25 by 4-bromobutyl acetate did not proceed smoothly probably due to its low solubility in solvent (DMF). The reaction of N2-acetyl-7-benzylguanine26, however, gave rise to N2-acetyl-7- benzyl-9-(4,acetoxybutyl)guanine27as a bromide salt in an $\mathbf{8 7 \%}$ yield. After completion of reaction, the product was easily obtained by simply adding ethyl acetate and filtering. Deprotection of the 7-benzyl group was achieved by catalytic hydrogenolysis in $\mathrm{MeOH}$ to give N2-acetyl-9(4-acetoxybutyl)guaninewhich was further treated with aqueous $\mathrm{NaOH}$ to give 9-(4hydroxybutyl)guanine 28 in a $94 \%$ yield (eq. 11). It is worthy of note that the product was not contaminated with the 7 -isomer at all. It was confirmed that the reaction sequence took place in a highly regioselective manner. Furthermore, it was found that the alkylation of $\mathbf{2 6}$ and the reductive removal of benzyl group proceeded in one pot. A similar reaction sequence using 2acetoxymethyl-4-iodobutylacetate afforded penciclovir 5 in a $75 \%$ yield in one isolated step from 26 (eq. 12).

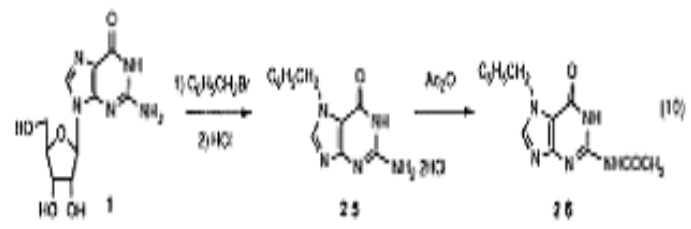

Conversion of 5 to famciclovir 6 was rather straightforward.5 was first acetylated with acetic anhydride and subsequently chlorinated with POCI3 to give the 6-chloro derivative 31. It should be noted that the chlorination of diacetylpenciclovir $\mathbf{3 0}$ went on more smoothly than that of guanine under similar conditions. The final transformation of $\mathbf{3 1}$ into famciclovir $\mathbf{6}$ was achieved in a highly efficient manner accordingto the reported procedure (eq. 13, Ref. 27). 


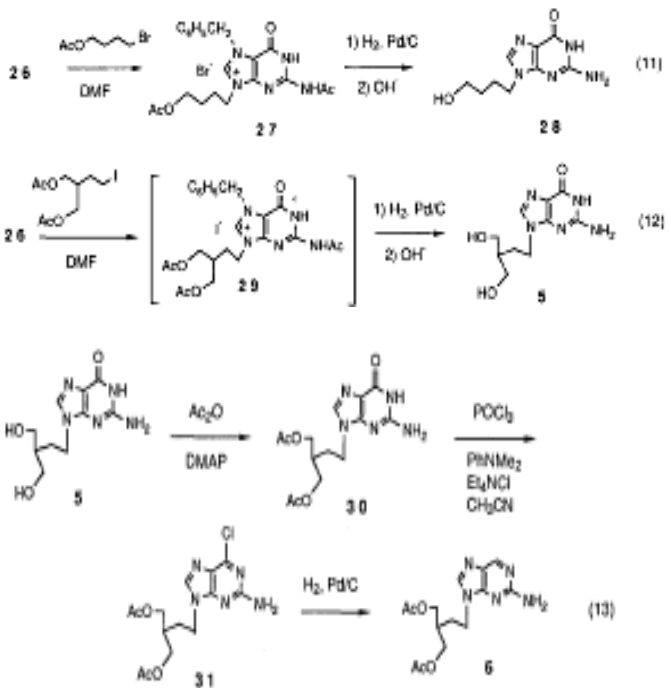

This approach should offer new routes to a variety of 9-substituted guanine and purine derivatives which are under development in several pharmaceutical companies. Chloroquine and Hydroxychloroquine and Emerging Viruses Chloroquine (CQ) is an aminoquinoline known since 1934. It was synthesized to be used as an antimalarial drug, but its properties and mechanism of action encouraged its use for the treatment of Microorganisms 2020, 8, 858 of 26 different diseases. Currently, CQ and its hydroxy-analog hydroxychloroquine (hydroxyCQ) cannot be used as antimalarial drugs in wide areas where the resistance of malaria parasites emerged. They are commonly used for connective tissue disorders, such as rheumatoid arthritis. Due to low toxicity and cost, high tolerability and immunomodulatory properties, CQ and hydroxyCQ have also been proposed for use against viral infections. Even if their specific mechanisms in individual diseases are not clear, it is well assessed that the antiviral activities of the aminoquinoline take advantage of their strong anti-inflammatory activity. The major proposed mechanisms of actions of $\mathrm{CQ}$ analogs which are suggested to influence the anti-viral activity are, among the others: the inhibition of cytokine production and release by T cells: IL-1, 2, 6, or 18, tumor necrosis factor TNF- $\alpha$ and IFN- $\gamma$, reduced levels of chemokines CCL2 and CXCL10, inhibition of micro-RNA expression, decreased TH17-related cytokines, decreased DNA, RNA and protein synthesis in thymocytes (reviewed in). The in vitro antiviral effect of CQ was first reported approximately 40 years ago, and since that time, its use as an antiviral drug has been extensively discussed. In particular, CQ/hydroxyCQ have been used for the treatment of emerging chikungunya virus (CHIKV) infection, recently causing numerous outbreaks in the world. Khan et al. showed that the treatment of infected Vero cells with different micromolar concentrations of $\mathrm{CQ}$ reduced virus yield and viral RNA copy number. De Lamballerie and colleagues confirmed the inhibition of CHIKV replication in Vero-E6 cells using CQ. The efficacy of CQ was inversely related to the concentration of the viral inoculum used, an unfavorable observation, considering the high viremia measured at the acute stage of CHIKV infection (up to 1010 virus copies/mL serum). Sourisseau and colleagues treated $\mathrm{HeLa}$ cells with CQ, obtaining a potent inhibition of CHIKV replication and its relative cytopathic effects. A double-blind placebo-controlled trial was designed to evaluate the efficacy and safety of CQ for the treatment of CHIKV infection in 2006 in French Reunion Island (Indian Ocean). No significant difference was observed between the CQ and placebo groups, either in the mean duration of febrile arthralgia or in the rate of viremia decrease. However, the number of patients included in the study was too small to draw definitive conclusions regarding the efficacy of CQ treatment Aminoquinolines were proposed for the treatment of other viral infections, such as ZIKV. In 2017, it was demonstrated that CQ and AQ exerted antiZIKV activity in Vero cells, with low micromolar IC50s. These results were in agreement with the decreasing number of ZIKV-infected cells after CQ treatment. Additionally, CQ protected the cells from further ZIKV infection, as measured by cell viability at noncytotoxic concentrations. The activity of CQ has also been indirectly demonstrated against DENV infection. The results obtained by Kleber and colleagues showed that CQ suppressed TNF- $\alpha$ and IFN- $\gamma$ production, and it was hypothesized that $\mathrm{CQ}$ might be used to treat patients suspected of having dengue disease, avoiding the more severe form of dengue hemorrhagic fever and/or shock. A clinical trial was also established to verify the effect of $C Q$ versus placebo in DENV-infected patients in Brazil. CQ promoted a reduction in the intensity of pain and an improvement in the well-being of patients with DENV infection but did not alter the duration of the disease or the intensity and days of fever To study the effects of CQ against EBOV, a group led by Dowall conducted an in vitro investigation using the human cell line MRC-5 and in vivo studies with the well-characterized guinea pig model. They were able to demonstrate that CQ reduced EBOV replication in MRC-5 cells. In contrast, the administration 
of CQ to 12 Guinea pigs did not protect the infected animals against the Ebola disease. Madrid and colleagues suggested that CQ could interfere with the late stages of EBOV replication and assembly. Despite these positive in vitro results, the clinical trials were sometimes conflicting. For this reason, later, the literature was reviewed to clarify the efficacy of CQ in the treatment of filovirus infection. It was concluded that the efficacy of CQ against the viruses belonging to this family was dependent on the CQ plasma concentrations, which must be sustained in patients until the clearance of the viremia . Microorganisms 2020, 8, 859 of 26 CQ was shown to inhibit the replication and spread of coronavirus ( $\mathrm{CoV})$ in vitro and to prevent infection with $\mathrm{CoV}$ in newborn mice. Since the suppressive effect of CQ was also present when the cells were treated before the infection, a prophylactic advantage of CQ use was suggested. 4.2. Chloroquine and Hydroxychloroquine and $\mathrm{HCV}$ CQ and its analogs have effects against HCV. In particular, the treatment of JFH-1 or Huh-7 cells with CQ reduced $\mathrm{HCV}$ entry, replication, and infection in a dose-dependent manner. Furthermore, CQ, in combination with IFN- $\alpha$, prevented the replication of $\mathrm{HCV}$ and enhanced the antiviral effect of IFN- $\alpha$. In this regard, two phase I/II clinical trials were initiated to verify the efficacy of the combination treatment of hydroxyCQ and ribavirin, but no results were posted due to limited recruitment. 4.3. Chloroquine and Hydroxychloroquine and HIV The anti-HIV-1 and anti-HIV-2 activities of CQ and its analogs were tested in vitro and in vivo. The first report about the in vitro use of CQ as an anti-HIV-1 agent was published in 1990 by Tsai et al., which showed the suppressive effects of CQ on the replication of HIV-1 in a T cell line. A few years later, Sperber and colleagues confirmed these results, showing the ability of CQ and hydroxyCQ to inhibit HIV-1 replication not only in $\mathrm{T}$ cells but also in monocytes. Subsequently, the same group demonstrated the CQ and hydroxyCQ anti-HIV-1 and anti-HIV-2 in vitro effects at concentrations that are clinically achievable. CQ had an additive effect against HIV-1 when used in combination with other antiretroviral agents. Naarding et al. demonstrated that CQ reduced HIV-1 transmission to and replication in CD4 $+\mathrm{T}$ lymphocytes. Similarly, Martinson et al. observed that CQ had a preventive role in HIV infection, reducing CD8 + T cell activation upon HIV replication. The antiviral activity of hydroxyCQ was demonstrated in vivo by several clinical trials. The somministration of hydroxyCQ was able to reduce the amounts of plasma HIV-1 RNA and IL-6 in patients treated for eight weeks compared to those of the placebo group . During a second clinical trial, hydroxyCQ was shown to reduce the HIV-1 RNA plasma level, although at a lower level than the antiviral zidovudine. In contrast, the results published in 2012 by Paton and colleagues showed negative results, with an increase in viral load and a decrease in CD4 number. Another double-blind, randomized placebo-controlled trial testing the effects of CQ chronically HIV-infected persons was conducted in Minnesota. The results showed that the administration of CQ during chronic HIV infection resulted in decreased immune activation, but no data regarding HIV status were reported . Very recently, the AIDS Clinical Trials Group A5258 was completed. It was a randomized, double-blind, placebo-controlled study in HIV-1-infected participants off antiretroviral therapy and participants on antiretroviral therapy. CQ modestly reduced immune activation in antiretroviral therapytreated HIV-infected participants. Finally, the recruitment of 1499 patients was concluded a few months ago in a randomized, controlled, open-label, phase III trial of the standard of care with CQ prophylaxis compared to no prophylaxis in HIV-positive patients in Malawi. Chloroquine and Hydroxychloroquine and Other RNA Viruses CQ was shown to inhibit the in vitro replication of $\mathrm{H} 1 \mathrm{~N} 1$ and $\mathrm{H} 3 \mathrm{~N} 2 \mathrm{IAV}$ strains. A phase II clinical trial aiming to verify the effect of CQ compared to that of placebo on IAV was started in Singapore in 2005, and 1516 patients were recruited. However, CQ was not shown to prevent infection with IAV. CQ had inhibitory effects on the entry and replication of enterovirus (EV)-A71 in cell-based systems. Yong and colleagues studied the efficacy of CQ against several EV serotypes and evaluated its therapeutic capacity in vitro in RD cells and in vivo in a murine model. They Microorganisms $2020,8,8510$ of 26 demonstrated the potential of CQ as an antiviral in the treatment of hand, foot, and mouth disease caused by EV infection. The positive results obtained in the murine model of infection were indicative of the fact that CQ may mitigate the disease severity in mammals. 4.4. Amodiaquine and Emerging Viruses Amodiaquine (AQ) was originally developed and has been widely used for the treatment of malaria. However, subsequent studies revealed that it was active against a wide range of human pathogens, including several 
viruses. In a study published in 2014 , it was investigated whether quinolone derivatives could inhibit the replication of DENV. The time-course analysis suggested that AQ was stable and that it reproducibly inhibited DENV infectivity. The data also showed that viral entry and internalization were partially inhibited by the drug, but the major effect occurred at a later stage of the viral life cycle. It is known that AQ inhibits EBOV replication in vivo. A recent study demonstrated that AQ was active against severe fever with thrombocytopenia syndrome (SFTS) caused by SFTS virus (SFTSV) . 4.5. Primaquine Primaquine was tested as an antiviral on primary chicken embryo cells (CECs) infected by Newcastle disease virus. It was demonstrated that primaquine had an effect on the accumulation of viral hemagglutinin on the cell surface. In addition, primaquine inhibited protein synthesis in virus-infected cells.

Dealing with suspected or confirmed H1N1 infection cases at different levels of care

We can easily notice that there are developed and from WHO and CDC recommended procedures in handling the diseased. In that sense from historical military sanitary doctrine it is well known that epidemiological surveillance and proper organisation on the field are much more important for raising survival rates than experienced physicians or quality of equipment available. Therefore, in accordance with this we present an algorythm on handling infectedpersons in primary care setting. Recommended procedure differs significantly at secondary and terciary levels of care and assumes selection and follow up of patients in line with natural course of disease and response to treatment. In order to more easily find the assistance on clinical decision making we gave the next algorythm No 2. The new strains will be in conditions of an increase in visitors, regardless of whether they have or not an immunologic experience with the previous strains. As a result of that, shortly after the appearance of a new type, the old forms will disappear and the new family will become dominant for a period which in general covers 10-20 years, in which there is, for the emergence of minor antigenic variation, the subdivision in various subtypes. The outcrops of a new epidemic strain may, therefore, be regarded as a process of development interesting the characteristics of the strain and the susceptibility of the population. In order that a strain has a wide distribution, its antigenic characteristics must ensure that it escapes the neutralization of antibodies of the host and of the surrounding population. So the outbreaks will happen with those strains that have dominant antigens that fit the deficiency, or better, the absences of antibody in the population. It seems, in conclusion, that the flu virus showes an ability and an aptitude for survival built on the possibility of emergence of new models that allow the virus being confused easily through populations still partly immune to previous antigenic forms

According to this view, the changes in the influenza A can be designed in single meaning, in the context of a principle and of an evolutionary progress, from Burnet said immunological drift or steering immunology. Very important to remember that it was demonstrated the presence of antibodies to the more recent strains of 1957 Asian flu (A2) in older segment of that population: in Asian influence there were obviously strains with dominant characters, other than those that had characterized the previous years, more or less, but similar to those of the strains widespread much before (1889-90pandemic).

For the emergency created by epidemic of avian flu in Asia it was right not to create panic as victims of a bad information (2). The possibility that the avian virus entries in other parts of the world it was like the rest for all types of flu viruses. It is clear that the dead animal is harmless, and therefore there are other veterinary and agricultural interests There is a potential risk of genetic recombination with human flu viruses that might hesitate to a viral variant capable of a transmission from human to human.

Some important medicinal natural herbs or plants used for the treatment of swine flu Basil: Ocimum sanctum. and Ocimum basilicum also known as Tulsi (Hindi) and Holy Basil (English), is an aromatic plant of the family Lamiaceae. The plant, as a whole, is a treasure house of potent compounds with its leaves, seeds, and roots, as well as flower being medicinally important and is considered divine by the Hindus. Ocimum sanctum. and Ocimum basilicum are great Ayurvedic treatment option for swine flu. Ayurvedic practitioners claim that basil not only keeps the nasty swine flu virus at bay, but it also assists in the fast recovery of an affected person. They claim that basil improves the body's overall defense mechanism, there by increasing its ability to fight viral diseases. It is 
also believed to strengthen the immune system of the afflicted person. For the control and prevention of many disease, Ocimum extracts are used in ayurvedic remedies for common colds, headaches, stomach disorders, inflammation, heart disease, various forms of poisoning, and malaria. For the control and prevention of swine flu, basil must be consumed in the fresh form. The paste or juice of a minimum of 25 leaves (medium size) should be consumed twice a day. Moreover, it should be had on an empty stomach. O. sanctum is considered to be an adaptogen par excellence (89). It harmonizes different processes in the body and is helpful in acclimatizing to stress. The main chemical constituents of O.sanctum are oleanolic acid, ursolic acid, rosmarinic acid, eugenol, carvacrol, linalool, and $\beta$-caryophyllene (10). O.sanctum is reported to be an effective treatment for diabetes and high cholesterol (11) O.sanctum also shows promise for protection against radiation damage (12-13). O.sanctum leaves contain highest percentage of essential oils, infusion of which is given in malaria. Juice of the leaves is taken internally and is very effective in skin diseases such as itches fungal infections. Fresh leaves also cure chronic fever and when mixed with honey and ginger juice, it is useful in cough and bronchitis (14). The antimicrobial properties of $\mathrm{O}$. sanctum make it useful for the prevention of novel H1N1 flu. Basil is safe, with no side effects and is great to prevent swine flu from spreading like wildfire

\section{HYDROXYCHLOROQUINE SULFATE}

\section{Manufacturing Process}

STAGE-I: Hydroxychloroquine Base: Charge 4,7-Dichloroquine,Hydroxynovaldiamine,

Sodium carbonate and Potassium iodide. Heat the RMto $125^{\circ} \mathrm{C}-130^{\circ} \mathrm{C}$ and maintain for $60-70$ hours. Cool the RM to RT. Extractthe RM in Ethyl acetate. Wash the Ethyl acetate layer with water. Acidify theRM with glacial Acetic acid to remove impurities. Then extract the RM inEthyl acetate at $\mathrm{pH}$ 12-13. Distill out solvent, charge Ethyl acetate to RM.Charcoalize the RM. Crystallize the Hydroxy chloroquine at $0^{\circ} \mathrm{C}-$ $10^{\circ} \mathrm{C}$.Hydroxy chloroquine base is again crystallizing from Ethyl acetate at $0^{\circ} \mathrm{C}-10^{\circ} \mathrm{C}$. Dry the wet hydroxy chloroquie base at $50^{\circ} \mathrm{C}-$ $55^{\circ}$ C.STAGE-II:

Hydroxychloroquine

Sulphate: Dissolve hydroxy chloroquine base in IPA and water. Charcoalize the RM at $70^{\circ} \mathrm{C}$ $80^{\circ} \mathrm{C}$. Charge aqueousSulphuric acid to raw material solution at $45^{\circ} \mathrm{C}-55^{\circ} \mathrm{C}$. Distill out 10$15 \%$ IPA.Filter the solid, wash with IPA. Dry the wet Hydroxychloroquine Sulphate at $85^{\circ} \mathrm{C}-$ $95^{\circ} \mathrm{C}$.

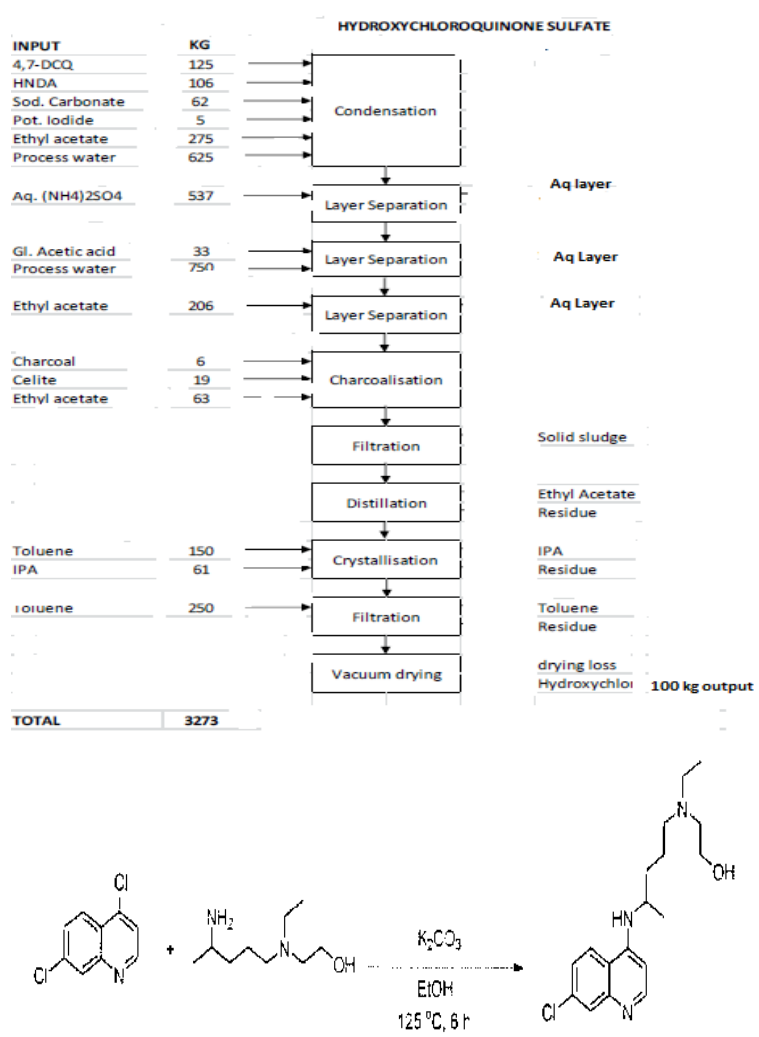

In the course of epidemic of avian influenza that struck in 200510 Asian countries (China, Pakistan, Thailand, Cambodia, Indonesia, North Korea, South Korea, Taiwan, Laos, Vietnam) with 80 million chickens died or sacrificed and 42 fatal human cases it was identified H5N1 as an etiologic agent, the same as the one that in 1997 had caused an epidemic outbreak in Hong Kong with 18 human subjects infected and 6 dead and with the sacrifice of 1.5 million chickens (3).

The Avian Influenza recent outbreaks with involvement of viral strains as H9N2 in 1999, infected two children and other individuals, and in 2003, infected a boy in Hong Kong, while H5N1 hit three subjects of a family killing two in 2003. At the same time in the Netherlands an epidemic from avian influenza viruses H7N7 hit 83 people and led to death a veterinarian.

In 2005 in the USA outbreaks of avian influenza have been identified in Texas and in Delaware (virus H7N2), and in the last State together with territories of Maryland and of Virginia there are working 14,000 people and 1,900 families that produce the $8 \%$ of the meat of American poultry, with a budget of one and a half billion dollars. In 2003 the American export in Europe has reached the share of eight million and eight hundred thousand eggs and 452 thousand 
chicks, respectively for 20 million and 3 million of Euro.

For the emergency created by the epidemic of "influence of the pigs" in Mexico it is correct not to create alarmisms being victims of a bad information (4). The possibility that the virus arrives in other parts of the world is real as for all the types of influence virus (5). For the SARS a direct contact was necessary, in practical terms the so-called droplets of Pflugge, for this swine influence it is different, in fact, it also spreads through the air to distance. And a potential risk exists of a panic syndrome that it often happens through a bad information or a scarce knowledge of the phenomenon. Then no alarmism because the number of the victims is decidedly inferior to other pandemics (6).Few years ago there was the announce in Naples of an outbreak of disease of Newcastle in a group of parrots coming from Pakistan. The disease of Newcastle represents an useful paradigm of the influence infection in man. Fortunately there is not a reported human pathology to this virus, for which the discovery of the outbreak of disease of Newcastle in Naples did not give worries of any sort for the health of the Neapolitans. Finally the risks of the disease of Newcastle are more tied to the breedings of home volatile that, not immune to this virus, can be exposed to the epidemic (7).

The vaccination against the influence is the most effective method to prevent the illness. From the moment that we find the isolation of a new flu virus, we must wait for the preparation of a new specific vaccine that will be ready for the next influence season in Autumn.

The antiviral drugs (inhibitors of the neuraminidasis, receptor of the virus surface) should be assumed within 48 hours by the appearance of the influence symptoms and the subjects that have had a close contact with people infected by the flu virus.

In conclusion The history of flu viruses teaches that influence originates from birds, usually aquatic, then it is transferred to man through the leap into pigs. The promiscuity of the herds, facilitates this transition and then the spread. Three pandemics caused by influenza A viruses, which occurred in the 20th century, have all had this origin: the 'Spanish flu' (1918, H1N1), the 'Asian flu' (1957, H2N2) and the 'Hong Kong flu' (1968, H3N2). The 2009 H1N1 influenza virus acted during the winter in Australia and New Zealand yielding a pattern effect for the treatment of patients during the winter in the
Northern Hemisphere. The performance of rapid diagnostic test for the detection of novel influenza A (H1N1) virus was evaluated by the Centers for Disease Control and Prevention.

In the absence of any vaccines to prevent COVID-19, there are many clinical trials (CT) taking place to find a treatment. These CTs are mainly focusing on either repurposing or repositioning the existing molecules. WHO has published a landscape of therapeutics which could be used for treating COVID-19, and some of them are undergoing CTs as well. Generally speaking, patents are not a concern when it comes to old molecules under CTs because these molecules are already out of patent protection.

However, a few of these molecules are still under patent protection in many countries. Two in particular - Remdesivir and Favipiravir - are under patent protection in India. The generic availability of these medicines can facilitate compassionate use and CTs in India without depending on supply from the patent holders. Therefore, the Government of India should use the compulsory licence or government use license to facilitate the generic production of these medicines.

Some of these medicines are very old molecules, such as chloroquine and hydroxychloroquine, and some are of very recent origin, such as the Lopinavir-Ritonavir combination, which is used to treat HIV/AIDS. Some of these new molecules are still under patent protection in many countries, which are currently using a part of CTs or compassionate use to treat COVID-19. Responding to the patent barriers, a few countries like Israel and Chile have issued compulsory licenses to allow a generic company to produce or use the patented medicine/invention without the permission of the patent holder. Germany even amended its patent law to facilitate the quick issuance of compulsory licenses, while Canada will do so soon. The national assembly of Ecuador passed a resolution empowering the health minister to issue these licenses.

Now, there is one patent granted to Remdesivir, and our search found another application is pending. The recently granted patent will expire only in 2035. Favipiravir is already part of five patents in India, although one of these patents has already expired. However, only a detail analysis can reveal 
whether existing patents block generic manufacturing.

Remdesivir is believed to be the most promising drug which can be used for treatment against COVID 19. It is a broadspectrum antiviral drug, which is currently undergoing clinical trials in China. The results are expected towards the end of April. The drug works by inhibiting the virus's ability to replicate itself inside cells. This indicates that the drug would effective even when a person is in the initial stage of infection and the virus is still reproducing in the upper respiratory tract.

The findings of severe respiratory disease concurrent with the circulation of H1N1 influenza was proved by the aforementioned test. Even the potential impact of pandemic influenza during the Hajj pilgrimage was taken in account to reduce the substantial effect on the crowd to spread the infection.

\section{REFERENCES}

[1] Zimmer SM and Burke DS. "Historical perspective --Emergence of influenza A (H1N1) viruses". New England Journal of Medicine 361.3 (2009): 279-285.

[2] Enserink M and Cohen J. "Virus of the year. The novel H1N1 influenza". Science326.5960 (2009): 1607.

[3] Tarro G and Esposito C. "Emerging viral agents at risk in global health approaches to early diagnosis and prompt therapy". International Conference on Bioinformatics and Computational Biology (BIOCOM'11). Las Vegas, Nevada, USA. CS REA Press (2011).

[4] Chowell G., et al. "Severe respiratory disease concurrent with the circulation of $\mathrm{H} 1 \mathrm{~N} 1$ influenza". New England Journal of Medicine

[5] 361.7 (2009): 674-679.

[6] Dawood FS., et al. "Emergence of a novel swine-origin influenza A (H1N1) virus in humans". New England Journal of Medicine 360.25

[7] (2009): 2605-2615.

[8] Lister P., et al. "Swine-origin influenza virus H1N1, seasonal influenza virus, and critical illness in children". Lancet 374 (2009): 605607.

[9] Morens DM., et al. "The persistent legacy of the 1918 influenza virus". New England Journal of Medicine361.3 (2009): 225-229.

[10] Esposito C., et al. "Tracking the 2009 H1N1 influenza virus in the Italian region Campania". Journal of Cellular Physiology227.7 (2012): 2813-2817.
[11] Swine. Custom Vaccines. Novartis. http://www. livestock.novartis.com/cv_swine.html

[12] Swine Flu: The predictable pandem-ic?". http://www.newscientist.com/article.

[13] Influenza Factsheet. Center for Food Security and Public Health, Iowa State University.

[14] http://www.cfsph.iastate.edu/Factsheets/pdfs/inf luenza.pdf.

[15] P.A. Levene and W.A. Jacobs. Chem. Ber. 44,746 (1911).

[16] Kuninaka. J. Agric. Chem. Soc., Japan. 34,489 (1960).

[17] T. Utagawa, H. Morisawa, T. Miyoshi, F. Yoshinaga, A. Yamazaki and K. Mitsugi. FEBS Lett. 109,261 (1980). b) H. Morisawa, T. Utagawa, T. Miyoshi, F. Yoshinaga, A. Yamazaki and K. Mitsugi. Tetrahedron Lett.21,47 (1980). c) T. Utagawa, H. Morisawa, F. Yoshinaga, A. Yamazaki, K. Mitsugi and Y. Hirose. Agric. Biol. Chem. 49, 1053 (1985). d) T. Utagawa, H. Morisawa, $\boldsymbol{S}$. Yamanaka, A. Ysmazaki, F. Yoshinaga and Y. Hirose. ibid. 49,2167,2711 (1985). e) T. Utagawa, H, Morisawa, S. Yamanaka, A. Yamazaki, F. Yoshinaga and Y. Hirose. ibid. 50, 121 (1986).

[18] T. Utagawa, H. Morisawa, S. Yamanaka, A. Yamazaki, F. Yoshinaga and Y. Hirosc. Agric. Biol. Chem. 49.32 (1985).

[19] H. Shiragami, Y. Irie, H. Shirae, K. Yokozeki and N. Yasuda. J. Org. Chem. 53,517 (1988) b) H. Shirae, K. Kobayashi, H. Shiragami, Y. hie, N. Yasuda and K. Yokozeki. Appl. Environ. Microbiol. 55,419 (1989).

[20] B. V. Joshi, T. Rao, R. Sudhakar, C.B. Reese. J. Chem. SOC. Perkin Trans. 1. 2537 (1992). b) N.D.P. Cosford and R.F. Schinazi. Nucleosides \&Nucleotides. 13, $101 \quad 1 \quad$ (1994) and REFerences cited therein.

[21] M. Ishii, H. Shirae and K. Yokozeki. Agric. Biol. Chem. 53, 3209 (1989).

[22] M.M. Mansuri, J.E. Starrett, Jr., J.A. Wos, D.R. Tortolani, P.R. Brodfuehrer, H.G. Howell and J.C. Martin. J. Org. Chem. 54, 4780 (1989).

[23] R. Marumoto and M. Honjo. Chem. Pharm. Bull. 22, 128 (1974).

[24] H. Huang and C.K. Chu. Synrh. Commun. 20, 1039 (1990).

[25] M.J. Robins, F. Hansske, N.H. Low, J.I. Park. Tetrahedron Lett.25, 367 (1984). b) Y. Amino and H. Iwagami. Chem. Pharm. Bull. 39,622 (1991).

[26] H. Shiragami, T. Ineyama, Y. Uchida and K. Izawa. Nucleosides \&Nucleotides. 15,47 (1966).

[27] H. J. Schaffer, L. Bcauchamp, P. de Miranda, G.B. Elion, D.J. Bauer and P. Collins. Nature (London). 272,583 (1978). b) K.K. Ogilivie, H.R. Hanna, N. Nguyen-ba and K.O. Smith. 
Nucleosides \&Nucleotides. 4, 507 (1978). c) J. R. Barrio, J.D. Bryant and G.E.Keyser. J. Med. Chem. 23, 572 (1980). d) M.J. Robins and P.W. Hatfield. Can. J. Chem. 60, 547 (1982).

[28] H. Matsumoto, C. Kaneko, K. Yamada, T. Takeuchi, T. Mori and Y. Mizuno. Chem. Pharm. Bull. 36, 1153 (1988).

[29] J. Boryski and B. Golankiewicz. Nucleosides \&Nucleotides. 6,385 (1987) and 8,529 (1989).

[30] H. Shiragami, Y. Koguchi, Y. Tanaka, S. Takamatsu, Y. Uchida, T. Ineyama and K. Izawa. ibid. 14,337 (1995).

[31] J.C. Martin, C.A. Dvorak, D.F. Smee, T.R. Mattews and J.P.H. Verheyden. J. Med. Chem. 26, 759 (1983). b) U.K. Pandit, W.F.A. Grose, T.A. Eggelte. Synth. Commun. 2, 345 (1972). c) M.R. Hamden, R.L. Jarvest, M.R. Boyd, D. Sutton and A. V. Hodge. J. Med. Chem. 32, 1738 (1989). d) A. Larsson, B. Oberg, $S$. Alenius, C.-E. Hagberg, N.G. Johansson, B. Lindborg, G. Stening. Antimicrob. Agents Chemother. 23, 664 (1983).

[32] G. Geen, T.J. Grinter, P.M. Kincey and R.L. Jarvest. Tetrahedron.46,6903 (1990). b) J. Kjellberg and N.G. Johansson. Nucleosides \&Nucleotides. 8,225 (1989).
[33] Ramirez A, Capuano AW, Wellman DA, Lesher KA, Setterquist SF, Gray GC (June 2006). Preventing Zoonotic Influenza Virus Infection. Emerging Infect. Dis., 2006; 12 (6): 996-1000.

[34] Q \& A: Key Facts About Swine Influenza (Swine Flu) - Diagnosis". Centers for Disease Control and Prevention. 24 April 2009. http://www.cdc.gov/swineflu/key_facts.html

[35] Chlorine Bleach: Helping to Manage the Flu Risk". Water Quality \& Health Council. April 2009.

[36] Self protection measures". LHC. http://pn. lifehugger.com/doc/989/Swine_Flu.

[37] WHO. Clinical management of human infection with pandemic (H1N1) 2009: revised guidance 2009. Availablefrom:http:// www. who.int/csr/resources/publications/swineflu/clin ical_management_h1n1.pdf

[38] WHO. Clinical management of human infection with pandemic (H1N1) 2009 virus infection, nterim

[39] Guidance from Expert Consul-tation 2009. Available from: http://www.emro.Who.int/ CSR/h1n1/pdf/h1n1_clinical_management.pdf

Citation: Dr Krishnasarma pathy, "Antiviral Nucleosides-Flu Viruses-Quinolines-COVID-19", International Journal of Research Studies in Medical and Health Sciences. 2020; 5(8): 14-25.

Copyright: ( 2020 Dr Krishnasarma pathy, This is an open-access article distributed under the terms of the Creative Commons Attribution License, which permits unrestricted use, distribution, and reproduction in any medium, provided the original author and source are credited. 